\title{
Rejection of artifact sources in magnetoencephalogram background activity using independent component analysis
}

\author{
Javier Escudero*, Roberto Hornero, Member, IEEE, Daniel Abásolo, Member, IEEE, Jesús Poza, \\ Alberto Fernández, and Miguel López, Member, IEEE
}

\begin{abstract}
The aim of this pilot study was to assess the usefulness of independent component analysis (ICA) to detect cardiac artifacts and power line interferences in magnetoencephalogram (MEG) recordings. We recorded MEG signals from six subjects with a 148-channel whole-head magnetometer (MAGNES 2500 WH, 4D Neuroimaging). Epochs of 50 s with power line noise, cardiac, and ocular artifacts were selected for analysis. We applied a statistical criterion to determine the number of sources, and a robust ICA algorithm to decompose the MEG epochs. Skewness, kurtosis, and a spectral metric were used to mark the studied artifacts. We found that the power line interference could be easily detected by its frequency characteristics. Moreover, skewness outperformed kurtosis when identifying the cardiac artifact.
\end{abstract}

\section{INTRODUCTION}

$\mathrm{M}$ AGNETOENCEPHALOGRAM (MEG) recordings capture the neural activity in a non-invasive way by measuring the brain magnetic fields. This technique provides high temporal and spatial resolution, and it does not depend on any reference point [1]. In addition, magnetic fields are less distorted than electric ones by the skull [1]. However, MEG data are severely polluted by additive noise. In order to reduce this noise, MEG data are recorded in magnetically shielded rooms with low-noise SQUID gradiometers [1].

Unfortunately, external additive noise is not the only undesired signal in MEG data. Brain signals are mixed with other non-cerebral sources (i.e., artifacts) which could influence the analyses. The main artifact is the cardiac one, which shares its frequency band with the brain signals. Moreover, such a signal always appears in MEG recordings, and its amplitude is usually high enough to be visible in raw data [2]. As well as the cardiac artifact, the ocular blinks can also be evident in MEG recordings [3], and the power line interference may also be present in these data [1].

Several methods, such as epoch rejection, regression techniques [4], or principal component analysis [5], have been used to deal with different kinds of artifacts in

Manuscript received April 6, 2006. This work was partially supported by "Ministerio de Educación y Ciencia" and FEDER grant MTM 2005-08519C02-01 and a project from the "Consejería de Fomento de la Junta de Castilla y León". Asterisk indicates corresponding author.

J. Escudero, R. Hornero, D. Abásolo, J. Poza, and M. López are with the E.T.S. Ingenieros de Telecomunicación, University of Valladolid, Camino del Cementerio s/n, 47011-Valladolid, Spain (phone: +34 983 423000, ext. 5589; fax: +34 983 423667; e-mails: jescrod@ribera.tel.uva.es, \{roberto.hornero, daniel.abasolo, jesus.poza, miguel.lopez\}@tel.uva.es).

A. Fernández is with the Centro de Magnetoencefalografía Dr. PérezModrego, Universidad Complutense de Madrid, Spain (e-mail: aferlucas@med.ucm.es). electroencephalogram (EEG) and MEG recordings. Recently, independent component analysis (ICA) [6] has become popular to reject artifacts from EEG and MEG data [7]-[15], since it needs neither previous information nor orthogonality between artifacts and useful signals.

This use of ICA can be summarized as follows. First, ICA provides a "demixing" matrix which decomposes the MEG channels into different independent components (ICs). Next, the ICs that are responsible for artifacts are detected by a visual inspection [7] or by automatic methods [13]-[15], and they are removed. Finally, a "mixing" matrix is used to project back the sources to the MEG channels in true sign and amplitude without the removed artifacts.

A major problem with this artifact rejection method is the artifact sources recognition. A few studies have proposed methods to automatically accomplish this task. For instance, Delorme et al. [11] proposed the use of kurtosis and entropy to identify artifacts in EEG data. With the addition of a correlation measure to these statistics, Barbati et al. [14] developed a semi-automatic approach to detect various types of artifacts in MEG signals. Recently, three criteria related to amplitude thresholds, power in high frequency bands, and scalp distributions of the ICs were used by Ting et al. [15] to deal with muscular and ocular artifacts in EEG.

Another open issue is how to select the number of ICs. Whereas some papers set a power threshold [15], others studies used the number of available channels as the number of sources [12], [13]. However, few statistical criteria have been used [9], [16].

In this study, we have used a statistical criterion to determine the number of ICs. Then, we have applied a robust method to estimate those ICs. Criteria based on higher order statistics and spectral properties have been introduced to detect the cardiac and power line artifacts. We wanted to test whether this method is able to detach the cardiac artifact and the power line interference from MEG data.

\section{METHODS}

\section{A. Linear mixing model and ICA algorithm}

In the ICA model for stationary sources [9], [14]-[16], $n$ MEG channels, $\mathbf{x}(t)=\left[x_{1}(t), x_{2}(t), \ldots, x_{n}(t)\right]^{\mathrm{T}}$, are considered a linear mixture of $m$ ICs, s $(t)=\left[s_{1}(t), s_{2}(t), \ldots, s_{m}(t)\right]^{\mathrm{T}},(m \leq$ $n)$. The model may also include an $n$-dimensional vector of additive external spatially uncorrelated Gaussian noise, $\mathbf{v}(t)$. 
Mathematically:

$\mathbf{x}(t)=\mathbf{A} \mathbf{s}(t)+\mathbf{v}(t)$

where $\mathbf{A}$ is a full rank $n \times m$ mixing matrix, and $\mathbf{s}(t), \mathbf{x}(t)$, and $\mathbf{v}(t)$ are supposed to have zero mean.

In our problem, only the observations, $\mathbf{x}(t)$, are known. Hence, $\mathbf{A}, \mathbf{s}(t)$, and $\boldsymbol{\Psi}=\mathrm{E}\left\{\mathbf{v}(t) \mathbf{v}(t)^{\mathrm{T}}\right\}$, where $\mathrm{E}\{\cdot\}$ is the expectation value, have to be estimated blindly from $\mathbf{x}(t)$. Several assumptions must be taken to find $\mathbf{A}$ and $\mathbf{s}(t)$ with ICA: independent and non-Gaussian ICs, instantaneous linear mixing, and stationary data. These hypotheses have been extensively reviewed and validated for EEG and MEG data in several papers (e.g., [17], [18]). Under such assumptions, the ICs can be estimated using a demixing matrix, $\mathbf{W}=\hat{\mathbf{A}}^{+}$(apexes " + " and " " " denote a pseudoinverse matrix, and an estimated variable, respectively):

$$
\mathbf{y}(t)=\mathbf{W} \mathbf{x}(t)=\mathbf{W}[\mathbf{A s}(t)+\mathbf{v}(t)]=\hat{\mathbf{s}}(t)+\mathbf{W} \mathbf{v}(t),
$$

where $\mathbf{y}(t)=\left[y_{1}(t), y_{2}(t), \ldots, y_{m}(t)\right]^{\mathrm{T}}$ is an $m$-dimensional vector of the estimated ICs.

Since we have modeled the MEG recordings as noisy mixtures of ICs, the ICA algorithm must be robust to external noise. Thus, we have used the cumulant-based iterative inversion (CII) algorithm [19], which is implemented in the ICALAB package [20] under the name Equivalent Robust ICA. Although the recordings may be contaminated by external Gaussian noise, the CII algorithm is able to find an asymptotically unbiased estimation of $\mathbf{W}$ when calculations are carried out with enough samples (typically $\geq 5000$ ) [19].

\section{B. Robust preprocessing}

It is suitable to reduce the problem dimensionality before the CII algorithm is applied to the data. To perform this dimensionality reduction in noisy data, the preprocessing stage must be robust to the external noise, and the diagonal elements in $\boldsymbol{\Psi}$ have to be taken into account. Thus, we implemented this preprocessing stage using the unweighted least squares method [16] of factor analysis (FA) [21]. Furthermore, this method allowed us to estimate $\hat{\mathbf{\Psi}}$.

Let us define $\mathbf{C}$ as $\mathrm{E}\left\{\mathbf{x}(t) \mathbf{x}(t)^{\mathrm{T}}\right\}$, and $\hat{\mathbf{A}}_{\mathrm{Pr}}$ as the preprocessing mixing matrix that relates the preprocessed data, $\mathbf{z}(t)$, to $\mathbf{x}(t)$ :

$\mathbf{x}(t)=\hat{\mathbf{A}}_{\mathrm{Pr}} \mathbf{z}(t)+\mathbf{v}(t)$.

Briefly, $\hat{\mathbf{A}}_{\mathrm{Pr}}$ is calculated from the eigenvalue decomposition of $(\mathbf{C}-\hat{\mathbf{\Psi}})$ using an initial estimation of $\hat{\mathbf{\Psi}}$. Then, $\hat{\boldsymbol{\Psi}}$ is estimated as the diagonal elements of $\left(\mathbf{C}-\hat{\mathbf{A}}_{\mathrm{Pr}} \hat{\mathbf{A}}_{\mathrm{Pr}}^{\mathrm{T}}\right)$. Both steps are repeated until $\hat{\mathbf{\Psi}}$ and $\hat{\mathbf{A}}_{\mathrm{Pr}}$ reach stable values [16].
Once $\hat{\boldsymbol{\Psi}}$ and $\hat{\mathbf{A}}_{\mathrm{Pr}}$ had been estimated, we calculated the prewhitening matrix, $\mathbf{Q}$, as the pseudoinverse of $\hat{\mathbf{A}}_{\mathrm{Pr}}$ using the Barlett method [22], which helps to reduce the external noise when reconstructing the data:

$\mathbf{Q}=\left(\hat{\mathbf{A}}_{\mathrm{Pr}}^{\mathrm{T}} \hat{\mathbf{\Psi}}^{-1} \hat{\mathbf{A}}_{\mathrm{Pr}}\right)^{-1} \hat{\mathbf{A}}_{\mathrm{Pr}}^{\mathrm{T}} \hat{\mathbf{\Psi}}^{-1}$.

The preprocessed data are obtained as $\hat{\mathbf{z}}(t)=\mathbf{Q x}(t)$. This method assumes that $m$ is known. However, $m$ must also be estimated from the data. Taking into account the number of free parameters and $m \leq n$, we have the following bound for the $m$ integer [9]:

$m \leq m_{\max }=(1 / 2)(2 n+1-\sqrt{8 n+1})$.

In order to determine $m$, we used a method derived from FA and based on statistical model selection with information criteria. We estimated $\hat{\boldsymbol{\Psi}}$ and $\hat{\mathbf{A}}_{\mathrm{Pr}}$ for each $1 \leq m \leq m_{\max }$. Then, the minimum description length (MDL) was computed according to the formula given in [9] for each $m$ value. Finally, the number of sources is estimated as the $m$ value which minimizes the MDL.

\section{Methods to detect artifacts}

In order to detect the power line interference and the cardiac artifact, we used the following metrics.

1) Spectral characteristics of a power line signal

ICA can isolate most of the power line interference into an IC [10]. Hence, the spectrum of the IC that may contain the line noise should be centered at the power line frequency ( $50 \mathrm{~Hz}$ in Europe). Therefore, we calculate a spectral metric, $R_{50 \mathrm{~Hz}}$, which measures the fraction of the power spectral density (PSD) contained between $49.5 \mathrm{~Hz}$ and $50.5 \mathrm{~Hz}$ for each IC. The ICs with $R_{50 \mathrm{~Hz}}$ values larger than a threshold $\left(t h_{50 \mathrm{~Hz}}\right)$ are marked as power line artifacts.

2) Kurtosis and skewness to detect cardiac artifact

Let $m_{n}=\mathrm{E}\left\{(x-\mathrm{E}\{x\})^{n}\right\}$ be the $n$th central moment of a distribution. Kurtosis excess $(K r E)$ and skewness $(S k w)$ are defined as:

$$
\begin{aligned}
& K r E=\frac{m_{4}}{\left(m_{2}\right)^{2}}-3, \\
& S k w=\frac{m_{3}}{\left(m_{2}\right)^{3 / 2}} .
\end{aligned}
$$

$K r E$ is negative for "flat" amplitude distributions, whereas it is positive for "peaked" ones [11], [14]. On the other hand, $S k w$ measures the asymmetry degree of a distribution. Only if the distribution is symmetrical, $S k w$ is zero. Therefore, the maximum values of $K r E$ and $\operatorname{abs}(S k w)$ must be related to "peaked and asymmetrical" ICs amplitude distributions, which are typical of cardiac artifacts. 


\section{MEG recording}

MEG recordings were obtained from six elderly subjects without past or present neurological disorders - age $=63.3 \pm$ 5.7 years; mean \pm standard deviation $(\mathrm{SD})-$. All subjects gave their informed consent for the participation in this study, which was approved by the local ethics committee.

The subjects lay comfortably on a patient bed during the MEG recording. They were asked to stay awake with eyes closed and to reduce eye and head movements. For each subject, five minutes of MEG data were acquired at a sampling frequency of $678.17 \mathrm{~Hz}$ with a 148-channel wholehead magnetometer (MAGNES 2500 WH, 4D Neuroimaging) in a magnetically shielded room. The recordings were down-sampled to $169.549 \mathrm{~Hz}$ (50,863 data points). Twelve epochs of $50 \mathrm{~s}(8,477$ samples) with cardiac and ocular artifacts were selected from the MEG signals. These epochs were digitally filtered using a band-pass filter with cut-off frequencies at 0.5 and $60 \mathrm{~Hz}$.

\section{RESULTS}

Twelve epochs of $50 \mathrm{~s}$ were processed to remove the cardiac and power line interference.

First, we estimated the optimal value of $m$ for each MEG epoch. The averaged $m$ value was $29.7 \pm 8.0$ ICs (mean \pm SD). Considering these values of $m$, the preprocessing stage estimated that the external noise represented the $7.94 \pm 9.55$ $\%$ (mean $\pm \mathrm{SD}$ ) (minimum: $1.89 \%$; maximum: $28.83 \%$ ) of MEG recordings energy. If the estimated external noise energy was not considered, the retained ICs accounted for the $99.17 \pm 0.58 \%$ (mean \pm SD) of the signal energy.

Once we had preprocessed the epochs, we carried out the ICA decomposition. The estimated ICs were visually inspected in both time and frequency domains, and they were classified into different groups: cardiac artifacts, ocular artifacts, power line interferences, and other signals. In all epochs, one IC was responsible for the cardiac artifact, and another IC contained most of the power line interference.

First, we used a metric based on calculating the fraction of the signal PSD that is centered at $50 \mathrm{~Hz}$ in order to measure the weight of the power line interference in each IC. Setting $t h_{50 \mathrm{~Hz}}=0.50$, we could automatically detect all the main power line interferences. Fig. 1(a) illustrates this case. Whereas the minimum $R_{50 \mathrm{~Hz}}$ value in the 12 power line ICs was 0.58 , the maximum $R_{50 \mathrm{~Hz}}$ value in any other IC was lower than 0.05 , except for an IC that provided $R_{50 \mathrm{~Hz}}=0.15$.

Finally, we used two higher order statistics to detect the cardiac artifact. The $K r E$ and $\operatorname{abs}(S k w)$ values were calculated from each IC. The IC that provides the maximum value for each metric might be related to the cardiac artifact. The results showed that the $K r E$ metric was able to recognize correctly nine of the twelve ICs $(75 \%)$ that explained the cardiac signals. In the other three cases, $K r E$ pointed to ICs visually related to ocular artifacts. On the other hand, $S k w$ detected correctly the cardiac artifact in all cases. Fig. 1(b) and (c) show this situation. We can observe that both ocular and cardiac artifacts have "peaked"
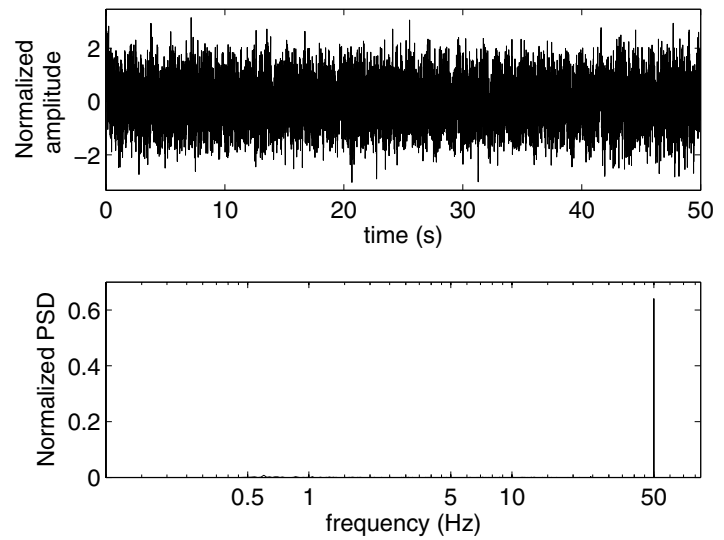

(a)
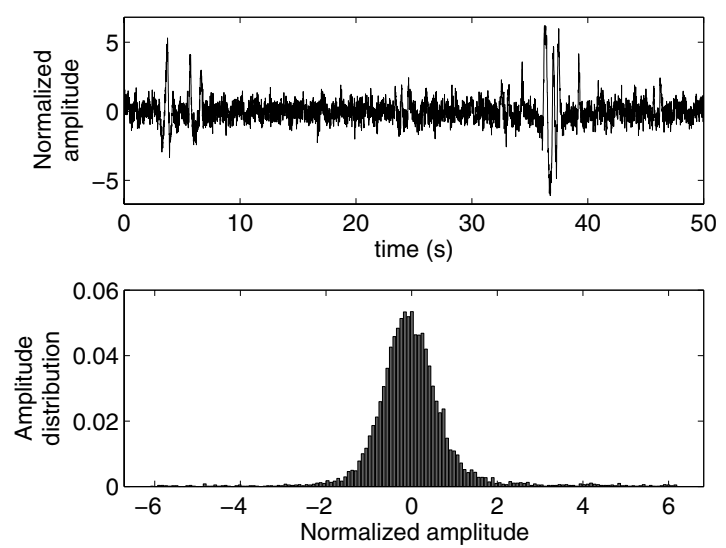

(b)
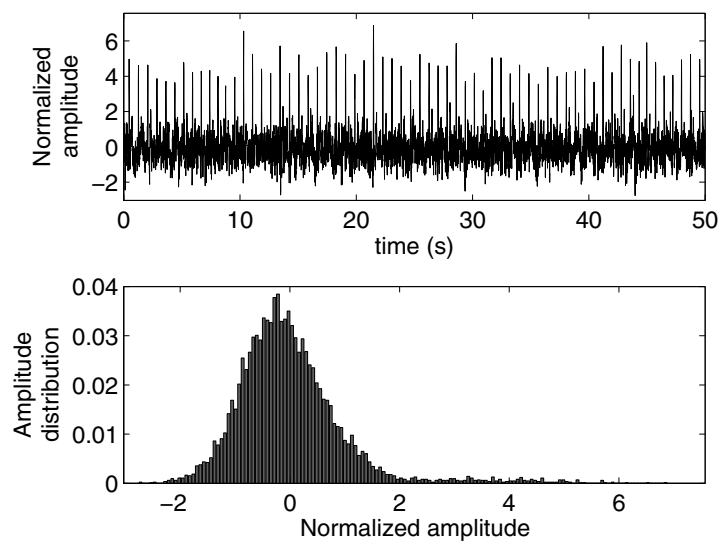

(c)

Fig. 1. Artifact detection by $R_{50 \mathrm{~Hz}}, K r E$ and $S k w$ on ICs from the same MEG epoch. (a) Power line artifact detected by $R_{50 \mathrm{~Hz}}\left(R_{50 \mathrm{~Hz}}=0.652\right.$; $K r E=-0.56 ; S k w=-0.006$ ). (b) IC wrongly marked as cardiac artifact by $K r E\left(R_{50 \mathrm{~Hz}}=0.002 ; \operatorname{Kr} E=10.17 ; S k w=0.856\right)$. (c) IC correctly marked as cardiac artifact by $S k w\left(R_{50 \mathrm{~Hz}}=0.003 ; \mathrm{KrE}=6.40 ; S k w=\right.$ 1.816).

amplitude distributions, which $K r E$ identifies easily. However, only the cardiac artifact has the asymmetrical distribution that $S k w$ detects.

\section{DiscusSION AND CONCLUSIONS}

MEG is a non-invasive neurophysiological technique that allows to measure the magnetic fields generated by brain 
activity with high temporal and spatial resolution [1]. However, MEG data are polluted by non-cerebral signals that could bias the analyses of the brain activity. To remove the cardiac and the power line artifacts, we have applied an ICA-based method. First, a robust preprocessing was applied [16], and a statistical criterion [9] was used to determine the number of ICs into which the data should be decomposed. Then, a robust ICA algorithm [19] found the ICs from the recordings. Finally, we evaluated the performance of different metrics to detect and remove those artifacts.

The MDL metric automatically selected the number of sources in which each MEG epoch should be decomposed. From the 148 available channels, the mean number of ICs was $29.7 \pm 8.0$. The averaged energy retained by these numbers of ICs was $99.17 \pm 0.58 \%$. This metric contrasts to other criteria used frequently in ICA-based studies (i.e., fixing the number of ICs equal to the number of EEG channels [12], [13], or setting a power threshold [15]).

To detect the ICs that account for the major power line interference, we calculated the fraction of the signal PSD centered at $50 \mathrm{~Hz}$. This metric is very easy and intuitive. Setting a threshold $t h_{50 \mathrm{~Hz}}=0.50$, we could detect all the power line artifacts. Moreover, we found that $R_{50 \mathrm{~Hz}}$ values clearly differentiated this kind of artifacts from any other IC. When detecting the cardiac artifact, we found that $S k w$ could detect artifacts that $K r E$ had missed. This could be explained by the fact that, whereas $K r E$ offers high values for "peaked" amplitude distributions such as the ocular or the cardiac signals, $S k w$ marks asymmetrical distributions, which are typical of the cardiac artifact.

Certain limitations of this study need to be considered. First, the sample size was small. Although the results show that our methods could be useful to recognize artifacts, further analysis must be carried out with a larger number of epochs and subjects. In addition, the interactions between our metrics and other kinds of artifacts (e.g., ocular or muscular ones) have to be studied. Second, evaluation of artifact removal is difficult since the true brain signals, without the influence of artifacts, are not known. Thus, a numerical index to automatically compare the original data (noisy recordings) and the reconstructed data (cleaned recordings) is lacking. Therefore, the improvement of the recordings quality is evaluated only by visual inspection.

To sum up, our analysis suggests that $S k w$ could be a useful metric to detect cardiac artifacts after an ICA algorithm estimates the ICs. We found that $S k w$ recognized the 12 ICs that were visually related to cardiac artifacts, whereas $\mathrm{KrE}$ only marked nine of them. Besides, the major power line interferences could be rejected by setting a threshold on the fraction of their PSD at $50 \mathrm{~Hz}$. However, the analysis must be extended to more MEG epochs with other kinds of artifacts to confirm these preliminary results.

\section{REFERENCES}

[1] M. Hämäläinen, R. Hari, R. J. Ilmoniemi, J. Knuutila, and O. V. Lounasmaa, "Magnetoencephalography - theory, instrumentation, and applications to noninvasive studies of the working human brain," Rev. Modern Phys., vol. 65, no. 2, pp. 413-497, Apr. 1993.

[2] V. Jousmäki, and R. Hari, "Cardiac artifacts in magnetoencephalogram," J. Clin. Neurophysiol., vol. 13, no. 2, pp. 172-176, Mar. 1996.

[3] A. Antervo, R. Hari, T. Katila, T. Ryhänen, and M. Seppänen, "Magnetic fields produced by eye blinking," Electroenceph. Clin. Neurophysiol., vol. 61, no. 4, pp. 247-253, Oct. 1985.

[4] R. J. Croft, and R. J. Barry, "Removal of ocular artifact from the EEG: a review," Neurophysiol. Clin., vol. 30, no. 1, pp. 5-19, Feb. 2000.

[5] P. K. Sadasivan, and D. N. Dutt, "SVD based technique for noise reduction in electroencephalographic signals," Signal Process., vol. 55, no. 2, pp. 179-189, Dec. 1996.

[6] P. Comon, "Independent component analysis, a new concept?," Signal Process., vol. 36, no. 3, pp. 287-314, Apr. 1994.

[7] R. Vigário, "Extraction of ocular artifacts from EEG using independent component analysis," Electroenceph. Clin. Neurophysiol., vol. 103, no. 3, pp. 395-404, Sep. 1997.

[8] R. Vigário, V. Jousmäki, M. Hämäläinen, R. Hari, and E. Oja, "Independent component analysis for identification of artifacts in magnetoencephalographic recordings," in Advances in Neural Information Processing Systems. Cambridge, MA: MIT Press, 1998, vol. 10, pp. 229-235.

[9] S. Ikeda, and K. Toyama, "Independent component analysis for noisy data - MEG data analysis," Neural Netw., vol. 13, no. 10, pp. 10631074, Dec. 2000.

[10] T. P. Jung, S. Makeig, C. Humphries, T. W. Lee, M. J. McKeown, V. Iragui, and T. J. Sejnowski, "Removing electroencephalographic artifacts by blind source separation," Psycophysiol., vol. 37, no. 2, pp. 173-178, Mar. 2000.

[11] A. Delorme, S. Makeig, and T. Sejnowski, "Automatic artifact rejection for EEG data using high-order statistics and independent component analysis," in Proc. 3rd International Workshop on ICA., San Diego, 2001, pp. 457-462.

[12] T. H. Sander, G. Wübbeler, A. Lueschow, G. Curio, and L. Trahms, "Cardiac artifact subspace identification and elimination in cognitive MEG data using time-delayed decorrelation," IEEE Trans. Biomed. Eng., vol. 49, no. 4, pp. 345-354, Apr. 2002.

[13] A. Flexer, H. Bauer, J. Pripfl, and G. Dorffner, "Using ICA for removal of ocular artifacts in EEG recorded from blind subjects," Neural Netw., vol. 18, no. 7, pp. 998-1005, Sep. 2005.

[14] G. Barbati, C. Porcaro, F. Zappasodi, P. M. Rossini, and F. Tecchio, "Optimization of an independent component analysis approach for artifact identification and removal in magnetoencephalographic signals," Clin. Neurophysiol., vol. 115, no. 5, pp. 1220-1232, May 2004.

[15] K. H. Ting, P. C. W. Fung, C. Q. Chang, and F. H. Y. Chan, "Automatic correction of artifact from single-trial event-related potentials by blind source separation using second order statistics only," Med. Eng. Phys., vol. 28, no. 8, pp. 780-794, Oct. 2006.

[16] J. Cao, N. Murata, S. Amari, A. Cichocki, and T. Takeda, "A robust approach to independent component analysis of signals with highlevel noise measurements," IEEE Trans. Neural Netw., vol. 14, no. 3, pp. 631-645, May 2003.

[17] R. Vigário, and E. Oja, "Independence: a new criterion for the analysis of the electromagnetic fields in the global brain?," Neural Netw., vol. 13, no. 8, pp. 891-907, Oct.-Nov. 2000.

[18] C. J. James, and C. W. Hesse, "Independent component analysis for biomedical signals," Physiol. Meas., vol. 26, no. 1, pp. R15-R39, Feb. 2005.

[19] S. Cruces-Alvarez, A. Cichocki, and L. Castedo-Ribas, "An iterative inversion approach to blind source separation," IEEE Trans. Neural Networks, vol. 11, no. 6, pp. 1423-1437, Nov. 2000.

[20] A. Cichocki, and S. Amari, Adaptive Blind Signal and Image Processing: Learning Algorithms and Applications, Wiley, 2002.

[21] R. Reyment, and K. G. Jöreskog, Applied factor analysis in the natural sciences, Cambridge: Cambridge University Press, 1993.

[22] M. S. Barlett, "Factor analysis in psychology as a statistician sees it," Proc. Uppsala Symp. Psychological Factor Analysis, no. 3, pp. 4-23, 1953. 